\title{
Retrograde Single Stapling Technique for Laparoscopic Ultralow Anterior Resection
}

\author{
Hidejiro Kawahara $^{a}$ Kazuhiro Watanabe $^{\text {a }}$ Takuro Ushigome $^{\text {a }}$ Rohta Noaki $^{a}$ \\ Susumu Kobayashi ${ }^{a}$ Katsuhiko Yanaga ${ }^{b}$ \\ a Department of Surgery, Kashiwa Hospital, Jikei University School of Medicine, Chiba, and \\ ${ }^{b}$ Department of Surgery, Jikei University School of Medicine, Tokyo, Japan
}

\section{Key Words}

Laparoscopic surgery · Anterior resection $\cdot$ Single stapling technique

\begin{abstract}
A highly effective surgical technique is required for laparoscopic low rectal transection without sufficient distal margins when ultralow anterior resection (ULAR) with a stapler is a technical challenge for laparoscopic surgery. We report a retrograde single stapling technique (RSST) for laparoscopic ULAR. Total mesorectal excision is performed laparoscopically. After the anal side of the tumor has been closed transanally, the distal line in the right lateral wall of the rectum is partially transected with laparoscopic coagulation shears (LCS). The distal line in the rectum is transected circularly with LCS after detecting the cutting part in the right lateral wall of the rectum laparoscopically. The end of the distal rectum is closed by a purse-string suture using 2-0 prolene transanally. Intestinal resection is performed extracorporeally through a suprapubic incision. After an anvil is placed in the proximal end of the colon over a purse-string suture, it is introduced to the anal canal transabdominally. The purse-string suture is tied to the anvil shaft before connecting it to the center shaft of the circular stapler; the instrument is then fired to create end-to-end anastomosis. For
\end{abstract}

the prevention of anastomotic leakage, a 24-french balloon catheter which decompresses the anastomosis is inserted through the anus.

Copyright $\odot 2010$ S. Karger AG, Basel

\section{Introduction}

The double stapling technique (DST) has been adopted worldwide for laparoscopic anterior resection. There are two kinds of DST procedures: the first is the original DST, which is a horizontal transection of the rectum followed by anastomosis with a circular stapler [1, 2]; the other is modified DST, which is a vertical transection of the rectum followed by anastomosis with a circular stapler [3-5]. In laparoscopic anterior resection, the former is mainly performed for tumors $>6 \mathrm{~cm}$ from the anal verge. The latter can be performed for tumors $4-6 \mathrm{~cm}$ from the anal verge [3]. As the laparoscopic stapler does not allow low rectal transection without sufficient distal margins, ultralow anterior resection (ULAR) with a stapler is not available for laparoscopic surgery [6]. We applied the retrograde single stapling technique (RSST) for laparoscopic ULAR, and herein report our initial results. 


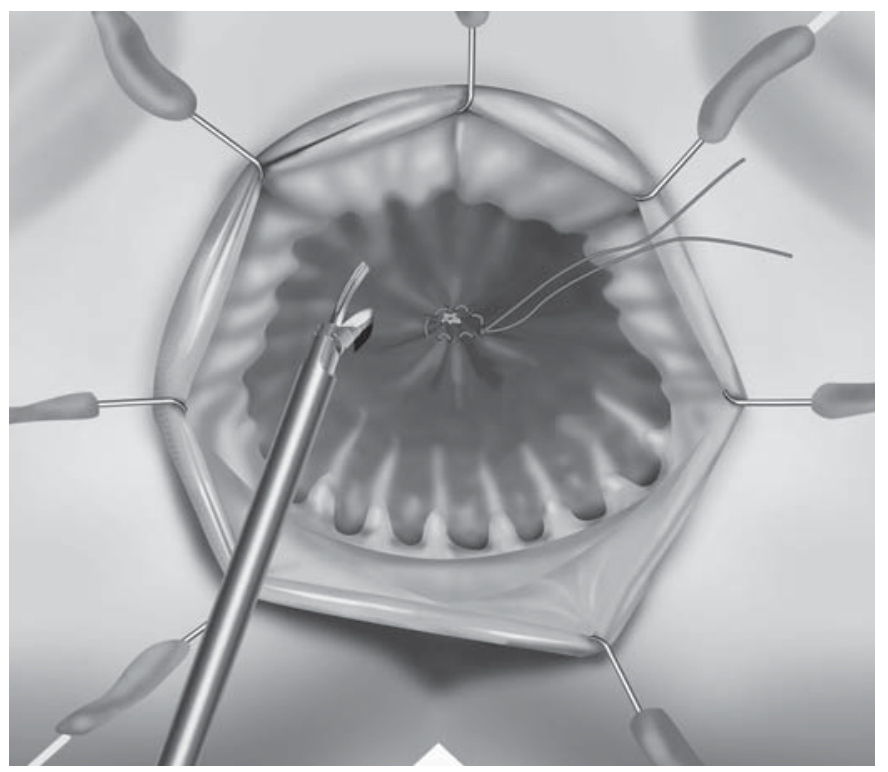

Fig. 1. After the anal side of the tumor is closed manually with a 2-0 prolene suture by a transanal approach, the distal line in the right lateral wall of the rectum is partially transected with LCS.

\section{Technique}

Patients are placed in the modified lithotomy position. Three trocars, $12 \mathrm{~mm}$ in diameter, are supraumbilically inserted into the abdominal cavity, in the upper- and lower-right quadrants of the abdomen. Two other trocars, $5 \mathrm{~mm}$ in diameter, are also inserted through incisions in the upper- and lower-left quadrants of the abdomen, respectively. Five trocars are used routinely, and occasionally another trocar is inserted through a suprapubic incision.

The superior rectal artery (SRA) is lifted up ventrally and the medial mesenteric attachments are incised. The superior hypogastric nerve plexus is preserved and separated dorsally from the SRA. Dissection is extended from the dorsal SRA to the left lateral attachment of the sigmoid colon. The mesorectum is separated along the plane of the total mesorectal excision $[7,8]$. To avoid damaging the visceral pelvic fascia, separation is continued to the 'holy plane' [9] and extended to the levator muscles.

The anal side procedure is started after the anus is sufficiently dilated by some retracting sutures or retracting instrument, such as the Lone Star Retractor. The rectal lavage is performed after the anal side of the tumor is closed manually with a 2-0 prolene suture by a transanal approach. The distal line in the right lateral wall of the rectum is partially transected with laparoscopic coagulating shears (LCS) (fig. 1). A gauze pad is inserted transanally to prevent air leakage during laparoscopic procedures. The distal line in the rectum is transected circularly with LCS after detecting the cutting part in the right lateral wall of the rectum laparoscopically (fig. 2). The end of the distal rectum is closed by a purse-string suture using 2-0 prolene transanally (fig. 3). Intestinal resection is performed extracorporeally through a suprapubic incision. After the placement of an anvil on the proximal end of the colon over a purse-string suture, the anvil was introduced to the anal canal transabdominally. The purse-string suture is tied to the anvil shaft before it is connected to

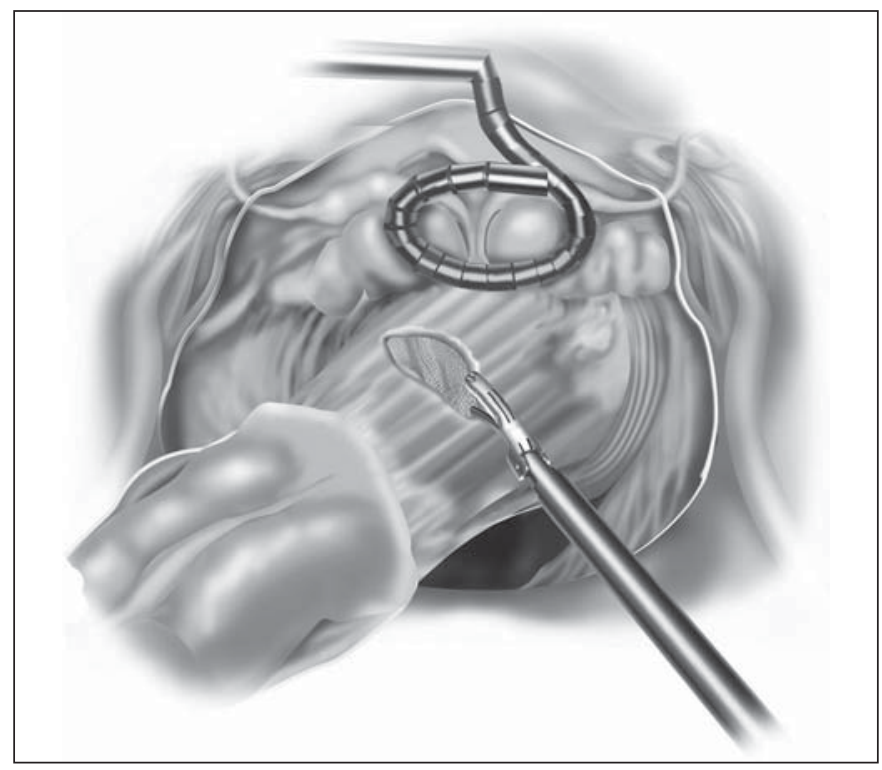

Fig. 2. The distal line in the rectum is transected circularly with LCS after detecting the cutting part in the right lateral wall of the rectum laparoscopically. the center shaft of the circular stapler, and the instrument is fired to create an end-to-end anastomosis (fig. 4). After the anastomosis, reinforcing sutures are placed manually if necessary.

Drains are inserted in two ways: one is the information drain that is inserted from the lower abdominal wall to the dorsal side of the anastomosis, and the other one prevents anastomotic leakage. A 24-french balloon catheter which decompresses the anastomosis is inserted through the anus (fig. 5) and removed 4 days after the operation. In the latter one, diverting ileostomy or colostomy is unnecessary.

\section{Results}

Laparoscopic ULAR with RSST is indicated for patients with a preoperative diagnosis of $\mathrm{T} 1$ or $\mathrm{T} 2$ carcinoma in the low rectum within $5 \mathrm{~cm}$ from the anal verge. Between January 2007 and December 2008, 5 patients (4 males, 1 female) with low rectal cancer underwent this procedure at our hospital. Their median age was 52 years (range 35-69). Median operation time was $200 \mathrm{~min}$ (range 165-250), intraoperative blood loss was $100 \mathrm{ml}$ (range 40-120), and postoperative hospital stay was 14 days (range 12-21). Postoperatively minor anastomotic leakage occurred in 1 patient; he did not require any surgical intervention and was discharged 21 days after the operation. None of the patients developed neurogenic bladder, and none of the 4 male patients developed any sexual disturbances. 


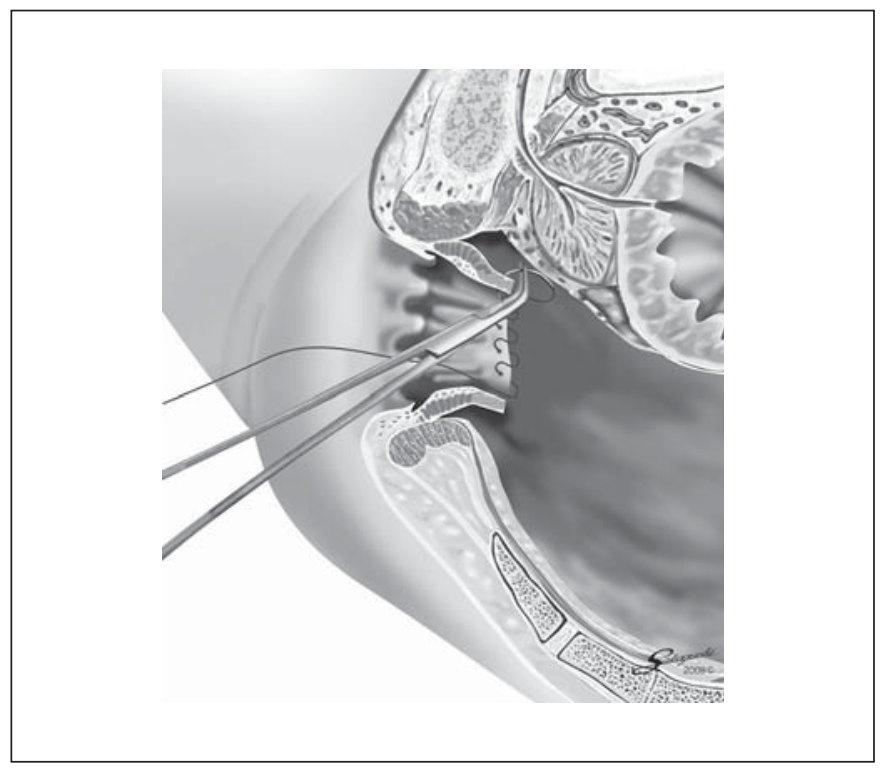

Fig. 3. A purse-string suture is placed transanally on the cut stump of the distal rectum.

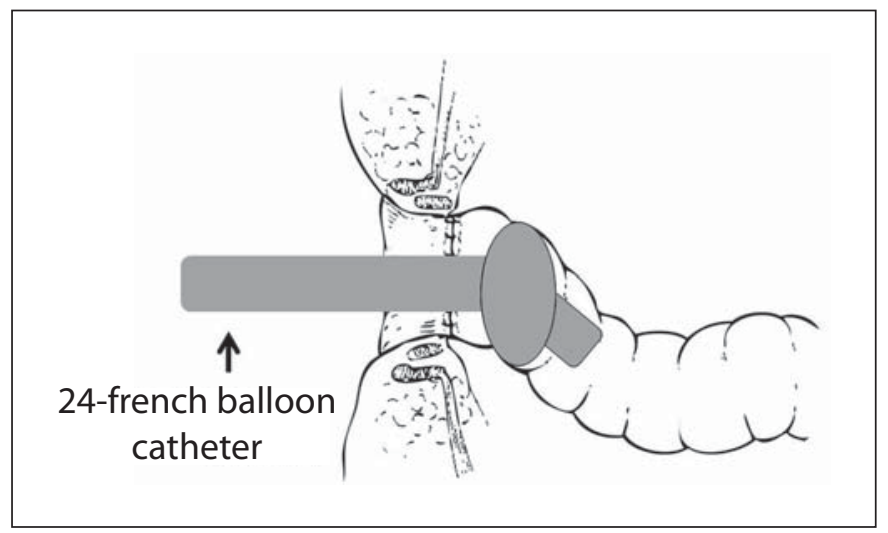

Fig. 5. After creating an end-to-end anastomosis by RSST, a 24 -french balloon catheter which decompresses the anastomosis is inserted through the anus to prevent anastomotic leakage.

\section{Discussion}

Although DST has been adopted worldwide for laparoscopic anterior resection, it is not indicated for ULAR. One of the most important reasons is the absence of adequate devices that allow safe transection of the rectum near the dentate line. Watanabe et al. [6] described per anum rectal resection and transanal manual anastomosis without autosuture devices in laparoscopic ULAR. How-

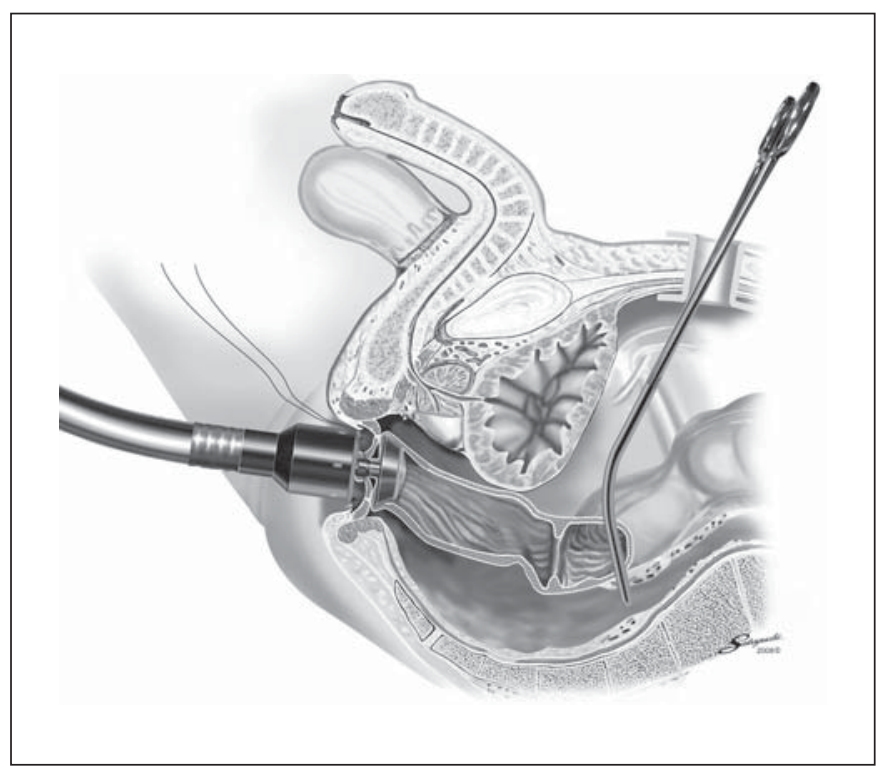

Fig. 4. After the anvil was introduced to the anal canal transabdominally, the purse-string suture is tied to the anvil shaft before connecting to the center shaft of the circular stapler.

ever, the procedure is complicated and requires expert skills. Fukunaga et al. [10] reported the prolapsing technique for laparoscopy-assisted low anterior resection with an auto-suture device. However, the rectum does not always allow easy eversion. On the other hand, laparoscopic ULAR with RSST can be completed quickly and allows simple and safe anastomosis. The single stapling technique is not indicated for tumors within $6 \mathrm{~cm}$ from the anal verge $[3,11]$, partly due to difficulty in placing a purse-string suture on the cut stump of the distal rectum. In this regard, RSST is a single stapling technique, but allows a purse-string suture to be placed transanally at the stump of the distal rectum.

Transecting the distal rectum by LCS may cause coagulation necrosis of the resection margin. To evaluate the resected doughnuts by anastomotic procedure in our patients, no necrosis is detected on the outside of the doughnuts in spite of detecting burned or necrotic tissue on the inside of them. If coagulation necrosis of the resection margin is made by the LCS procedure, it would be completely resected with a resected doughnut by circular stapler. Therefore, transecting the distal rectum by LCS would not greatly influence the anastomosis.

Although 1 of our 5 patients developed minor leakage, conservative treatment was possible. Because the anastomosis was located near the dentate line, intestinal contents were drained through the rectal balloon catheter 
which was inserted transanally during the operation. Transanal drainage prevents extension of inflammation to the abdominal cavity and promotes healing around the anastomosis. Needless to say that future examination is necessary, we believe that the transanal drainage will replace diverting ileostomy or colostomy.

\section{Conclusion}

Laparoscopic ULAR with RSST seems to be a safe and efficient procedure for selected cases with lower rectal cancer. The technique also allows secure division of the low rectum and reliable anastomosis.

\title{
References
}

1 Knight CD, Griffen FD: An improved technique for low anterior resection of the rectum using the EEA stapler. Surgery 1980;88: $710-714$.

2 Varma JS, Chan AC, Li MK, Li AK: Low anterior resection of the rectum using a double stapling technique. Br J Surg 1990;77:888-890.

- 3 Sato H, Maeda K, Hanai T, Matsumto M, Aoyama H, Matsuoka H: Modified doublestapling technique in low anterior resection for lower rectal carcinoma. Surg Today 2006; 36:30-36.

-4 Hamada M, Nishioka Y, Kurose Y, Nishimura T, Furukita Y, Ozaki K, Nakamura T, Fukui Y, Taniki T, Horimi T: New laparoscopic double-stapling technique. Dis Colon Rectum 2007;50:2247-2251.

5 Kawahara H, Hirai K, Suzuki T, Yoshimoto K, Kashiwagi H, Yanaga K: Mesorectum amputation technique for laparoscopic anterior resection. Int Surg 2007;94:235-238.

6 Watanabe M, Teramoto T, Hasegawa H, Kitajima M: Laparoscopic ultralow anterior resection combined with per anum intersphincteric rectal dissection for lower rectal cancer. Dis Colon Rectum 2000;43(suppl): S94-S97.

7 Heald RJ, Husband EM, Ryall RD: The mesorectum in rectal cancer surgery: the clue to pelvic recurrence? Br J Surg 1982;67:757-761.
-8 Enker WE: Potency, cure and local control in the operative treatment of rectal cancer. Arch Surg 1992;127:1396-1402.

-9 Heald RJ: The 'holy plane' of rectal surgery. J R Soc Med 1988;81:503-533.

$\checkmark 10$ Fukunaga M, Kidokoro A, Iba T, Sugiyama K, Fukunaga T, Nagakari K, Suda M, Yoshikawa S: Laparoscopy-assisted low anterior resection with a prolapsing technique for low rectal cancer. Surg Today 2005;35:598602.

$\checkmark 11$ Moritz E, Achleitner D, Holbing N, Miller K, Speil T, Weber F: Single versus double stapling technique in colorectal surgery. Dis Colon Rectum 1991;34:495-497.

\section{Invited Commentary}

\author{
H.-P. Bruch
}

Department of Surgery, Medical University of Lübeck, Lübeck, Germany

The authors describe a modified transection and stapling technique for ultralow carcinomas of the rectum which have been operated on by use of minimally invasive methods. Minimally invasive surgery follows the guidelines of open surgery. Features discerning minimally invasive surgery from open surgery are the mode of access, the gravity displacement of organs, and the magnification due to the signal transduction chain. The authors describe the transection of the inferior mesenteric artery and the sparing of the hypogastric plexus and nerves according to the guidelines. Neither do they transect the inferior mesenteric vein nor is the left flexure mobilized. This may lead to considerable stretch at the site of the anastomosis in very low anterior resections. In addition, it would be interesting to hear whether the left ureter is freed during the operation and whether Denonvilliers' fascia is left intact or resected.

The method of a transanal purse-string suture which is placed distally to the lowest tumor margin may be the only means to fulfill the principles of radicality and on- cological guidelines. Nevertheless, in our experience the transanal ultralow pull-through technique is only indicated in tumors reaching down to the sphincter apparatus or involving the upper part of the internal anal sphincter.

From our own experience we know that all other tumors can be resected transabdominally by use of a linear stapler if the pelvic floor is lifted by moderate cephalad pressure. In those cases presenting with a very low carcinoma of the rectum, the transanal approach which is described in the paper is the only means of curing the malignant disease and at least preserving parts of the internal anal sphincter. In very rare cases with an excellent external anal sphincter the total resection of the internal anal sphincter can be discussed with the patients.

The anastomosis can be carried out by use of a circular stapler as described by Hidejiro Kawahara and co-workers. A transanal suture technique can be performed with interrupted or running sutures if a Lone Star Retractor is inserted. 